\title{
Emergence of glassy-like dynamics in an orientationally ordered phase
}

\author{
M. Romanini, ${ }^{1}$ Ph. Negrier, ${ }^{2}$ J. Ll. Tamarit,,${ }^{1, *}$ S. Capaccioli, ${ }^{3}$ M. Barrio, ${ }^{1}$ L. C. Pardo,${ }^{1}$ and D. Mondieig ${ }^{2}$ \\ ${ }^{1}$ Grup de Caracterització de Materials, Departament de Física i Enginyeria Nuclear, ETSEIB, Diagonal 647, \\ Universitat Politècnica de Catalunya, 08028 Barcelona, Catalonia, Spain \\ ${ }^{2}$ Laboratoire Onde et Matière d' Aquitaine, UMR 5798 au CNRS-Université Bordeaux I, 351 cours de la Libération, \\ 33405 Talence Cedex, France \\ ${ }^{3}$ Dipartimento di Fisica, Università di Pisa, Largo B. Pontecorvo 3, I-56127 Pisa, Italy
}

(Received 27 September 2011; revised manuscript received 14 March 2012; published 4 April 2012)

\begin{abstract}
The dynamics of a simple rigid pseudoglobular molecule (2-adamantanone) has been studied by means of dielectric spectroscopy and examined under the constraints imposed by the space group of the crystal structure determined by $x$-ray powder diffraction. The low-temperature monoclinic structure of 2-adamantanone, with one molecule per asymmetric unit $\left(Z^{\prime}=1\right)$, displays a statistical intrinsic disorder, concerning the site occupancy of the oxygen atom along three different sites. Such a physically identifiable disorder gives rise to large-angle molecular rotations which inherently lead to time-average fluctuations of the molecular dipole, thus contributing to the dielectric susceptibility. The dielectric spectra for the low-temperature "ordered" phase displays a universal feature of glassy-like materials, i.e., coexistence of $\alpha$-and $\beta$-relaxation processes. The former is clearly identified with the strongly restricted reorientational motions within the long-range "ordered" crystalline lattice. The latter, never observed before in fully translationally and highly orientationally ordered phases, displays all the properties of an original Johari-Goldstein $\beta$-relaxation, in spite of the strong character of this glass-like phase. These findings can be explained according to the coupling model, applied to such "ordered" phases.
\end{abstract}

DOI: 10.1103/PhysRevB.85.134201

PACS number(s): 64.70.kj, 61.43.Fs, 76.60.-k, 81.05.Kf

\section{INTRODUCTION}

One of the major challenges in condensed matter physics concerns the dynamics of amorphous systems. ${ }^{1-3}$ Although the glass transition temperature, $T_{g}$, is associated with the primary $(\alpha-)$ relaxation process [at a relaxation time $\tau\left(T_{g}\right)=$ $100 \mathrm{~s}$ ] many other experimental features characterizing the dynamics of the amorphous systems appear. Among them, the emergence of secondary relaxations, especially of the so-called Johari-Goldstein (JG) $\beta$-relaxation, has attracted the attention of the large scientific glass community. ${ }^{4-13}$ This JG process is associated with local, noncooperative motions of the molecular unit as a whole, thus different from secondary relaxations due to intramolecular degrees of freedom (e.g., side-chain motions in polymers) and indeed it was found even in single rigid molecules. ${ }^{4,12}$ Despite the large quantity of available experimental and simulations studies, the nature of the local JG relaxation is still far from being understood. According to the original work, ${ }^{4,12} \beta$-relaxation appears as a consequence on the nonuniformity of the glassy state (islands of mobility) involving only local regions in which molecules can diffuse. An alternative homogeneous explanation attributes the secondary relaxation phenomena to small-angle reorientations of all the molecules. ${ }^{14}$

Although the JG relaxation is believed to be present in all types of glass formers, and thus sometimes thought as a precursor of the $\alpha$-relaxation, for many "strong" glasses (according to the "strong/fragile" Angell classification ${ }^{15}$ ) it has not been observed either as a clear peak ${ }^{6}$ or as a shoulder ${ }^{16}$ on the imaginary part of the dielectric susceptibility. The possibility that the characteristic relaxation time for the JG relaxation is not much shorter than that of the $\alpha$-relaxation cannot be ruled out. ${ }^{6,8,9,17-19}$ On the contrary, "fragile" glasses exhibit generally a distinct JG relaxation. It is worth recalling that some models even claim the nonexistence of the JG relaxation for strong glasses. ${ }^{20,21}$ In spite of the theoretical prediction that "amorphous packing" is the cause of the ubiquity of JG secondary relaxation in disordered systems, ${ }^{4}$ a new universal mechanism for this process has been recently proposed, related to the existence of dynamic heterogeneity and of molecular motions on different length scales ${ }^{22}$ and thus applicable, in principle, also to structurally ordered systems. Because no ultimate theoretical model for the glassy dynamics has been proposed so far, indeed experimental studies in simple systems allowing for the extraction of some fundamental features may be of particular importance.

In addition to the canonical glasses, obtained from supercooled liquids in which both translational and orientational degrees of freedom are frozen into disorder, orientational glasses (OGs) can be obtained as the nonergodic state of an orientationally disordered (OD) phase or plastic phase. ${ }^{23,24}$ This phase is characterized by the existence of a regular high-symmetry lattice formed by the centers of mass of the (pseudoglobular) molecules which are disordered with respect to the orientational degrees of freedom. This situation decreases the degree of complexity which is present in canonical glassy systems. ${ }^{25,26}$

By decreasing still more the complexity of the system, the emergence of the $\alpha$ - and $\beta$-relaxation scenario was found even in systems with ordered phase and ascribed to a new microscopic mechanism. ${ }^{27}$ It concerns tetrahedral molecules $\left(\mathrm{CBr}_{n} \mathrm{Cl}_{4-n}, n=0,1,2\right)$ forming a low-symmetry lattice that are nonequivalent with respect to their molecular environment. More specifically, the monoclinic low-temperature ordered phases of such materials contain $Z=32$ molecules with an asymmetric unit formed by $Z^{\prime}=4$ molecules and an intrinsic disorder with respect to the occupancy of the halogen sites for molecules with $n=1$ and $n=2$. The different dynamics exhibited by the molecules of the asymmetric unit give rise to a 
slower $(\alpha-)$ and a faster $(\beta-)$ relaxation, the slower one reaching a characteristic time of $100 \mathrm{~s}$ at $90 \mathrm{~K}$ and thus engendering the inherent glassy state associated with a peculiar disorder. It is thus evident that different scenarios giving rise to the emergence of secondary relaxations are possible so then "there seems to be no universal microscopic mechanism describing them." 27

The present work discusses the dynamics of an orientationally ordered (OO) low-temperature phase displaying statistical disorder, so yet another case for which the secondary relaxation could be ascribed to a different physical origin. The material studied is 2-adamantanone $\left(\mathrm{C}_{10} \mathrm{H}_{14} \mathrm{O}\right.$, hereinafter called $2 \mathrm{~A}=\mathrm{O})$. Adamantane derivatives display a rich polymorphism that has stimulated a large number of experimental and theoretical investigations in order to rationalize the dynamics of the high-temperature orientationally disordered (OD) phases and, to a lesser extent, the low-temperature OO phases. ${ }^{28-31}$ $2 \mathrm{~A}=\mathrm{O}$ is a "rigid" pseudoglobular molecule of $C_{2 v}$ symmetry obtained from adamantane by means of the substitution of two hydrogen atoms by one oxygen atom linked to a carbon by a double bond. Although the substitution of the oxygen atom gives rise to a strong dipole moment $(\mu=3.4 \mathrm{D})^{32,33}$ along the twofold symmetry axis, this compound exhibits still an OD high-temperature phase with face-centered-cubic $(F m 3 m)$ symmetry appearing below the melting point at $529 \mathrm{~K} .{ }^{23,33-35}$ This OD phase I transforms to an OO phase II at about $180 \mathrm{~K}$. Due to high hysteresis, the transition is found at $205 \mathrm{~K}$ on heating from the stable phase II.

The dynamics of the OD phase has been fully rationalized. Molecules in the fcc phase exhibit reorientational motions of the dipolar axis $\left(C_{3}\right)$ along the six fourfold $\langle 001\rangle$ cubic lattice directions, while around the $\mathrm{C}=\mathrm{O}$ axis of the molecules can display two positions related by a $\pi / 2$-rotation. ${ }^{34}$ As far as the low-temperature phase II is concerned, NMR experiments concluded that "all the motions are completely frozen." 35 Nevertheless, one of the reference works concerning the dynamics of OD phases ${ }^{23}$ by dielectric spectroscopy stated that the I to II phase transition only involves a "partial restriction of reorientational motion" which is accompanied with a strong slowing down of $\alpha$-relaxation time. Because dielectric spectroscopy is sensitive to the dipole fluctuations (and "blind" to the $\pi / 2$-rotation around the $\mathrm{C}=\mathrm{O}$ axis), such an $\alpha$-relaxation should involve orientational disorder of the dipolar molecules, in strong contradiction to the NMR results. In addition, astonishingly enough, Brand et al. indicated, within phase II, the emergence of a shoulder in the highfrequency wing of the $\alpha$-relaxation peak, possibly originated by a secondary $\beta$-relaxation.

\section{EXPERIMENTAL DETAILS}

In order to disentangle the dynamics of the low-temperature phase II of $2 \mathrm{~A}=\mathrm{O}$, we have combined $\mathrm{x}$-ray diffraction with broadband dielectric spectroscopy, extended to low temperatures. The compound was purchased from Aldrich Chem. Co., Inc., with purity of $99 \%$ and used as received.

High-resolution x-ray measurements were performed on a transmission mode diffractometer using Debye-Scherrer geometry (monochromatic $\mathrm{CuK} \alpha 1, \lambda=1.5406 \AA$, radiation) equipped with a horizontally mounted INEL cylindrical position-sensitive detector (CPS-120). Experimental details of the calibrations can be found elsewhere. ${ }^{36} \mathrm{X}$-ray patterns on a powder sample sealed in a $0.3-\mathrm{mm}$ diameter Lindemann capillary were obtained as a function of temperature (from 90 to $400 \mathrm{~K}$ ) by means of a 600 series cryostream cooler from Oxford Cryosystems and the large thermal hysteresis for the I-II transition was also revealed.

The relaxation times were measured by means of broadband dielectric spectroscopy conducted with a Novocontrol analyzer $\left(10^{-2}\right.$ to $\left.10^{7} \mathrm{~Hz}\right)$ and an HP4291 impedance analyzer $\left(10^{6}\right.$ to $1.8 \times 10^{9} \mathrm{~Hz}$ ), equipped with a Quatro temperature controller $( \pm 0.1 \mathrm{~K})$ and a closed-cycle helium cryostat. The capacitor was prepared by pressing the $2 \mathrm{~A}=\mathrm{O}$ powder between two stainless steel disks using a hydraulic press $(120 \mathrm{kN})$.

\section{RESULTS AND DISCUSSION}

$\mathrm{X}$-ray powder diffraction measurements were conducted from 90 to $205 \mathrm{~K}$ within phase II and from 210 to $400 \mathrm{~K}$ for OD phase I. The latter was easily indexed as face-centered cubic and due to the orientational disorder (the molecules jump discontinuously between a set of different orientation ${ }^{37}$ ) structural analysis was restricted to a Pawley profile fitting procedure.

As for the low-temperature phase II, potential solutions of the cell parameters and space groups were obtained at $190 \mathrm{~K}$ using X-Cell software available in the module Powder Indexing of Materials Studio. The cell parameters and space group compatible with the experimental $\mathrm{x}$-ray diffraction pattern were initially determined using a Pawley profile-fitting procedure which confirmed the initial indexing and the space group through the systematic absences. To fully characterize the low-temperature phase II of $2 \mathrm{~A}=\mathrm{O}$ a rigid body molecule was built up based on similar adamantane derivatives. ${ }^{38}$ Within the rigid body constraint, the structure of phase II of $2 \mathrm{~A}=\mathrm{O}$ was submitted to a Rietveld refinement. As an example, the experimental collected and final calculated x-ray profiles at $190 \mathrm{~K}$ are shown in Fig. 1.

The structure of $\mathrm{OO}$ phase II of $2 \mathrm{~A}=\mathrm{O}$ consists of a monoclinic $P 2_{1} / c, Z=2$, with lattice parameters $a=$ 6.5915(8) $\AA, b=11.1194(26) \AA, c=12.5891(13) \AA$, and $\beta=118.87(1)^{\circ}$ at $190 \mathrm{~K}$ and one molecule per asymmetric unit $\left(Z^{\prime}=1\right)$. The most striking feature of the structure of OO phase II is the existence of a statistical intrinsic disorder concerning the site occupancy of the oxygen atom. As can be seen in the left panel of Fig. 2 [(001) crystallographic plane], three possible sites are occupied by the oxygen atom with temperature-independent fractional occupancies of $25 \%$ $(\mathrm{O} 1$ and $\mathrm{O} 2)$ and $50 \%(\mathrm{O} 3)$. Within this "ordered" structure with $Z=2$ molecules per unit cell the symmetry operations according to the $P 2_{1} / c$ space group impose the disorder of the oxygen atom site with the aforementioned fractional factors. It means then that for each unit lattice, the oxygen atom of the asymmetric unit formed by one molecule $\left(Z^{\prime}=1\right)$ cannot be uniquely placed in one site but it shares three different sites (with different probabilities of occupancy).

It should be emphasized that the different but well-defined probabilities of site occupancies (i.e., fractional occupancies) together with the different angles between the three different molecular (and thus dipole) orientations make clear the 


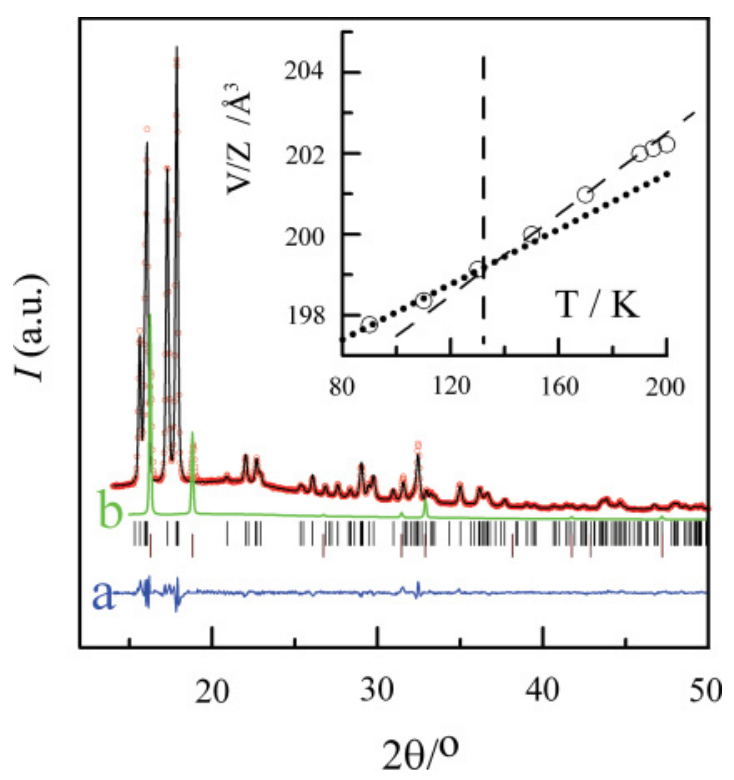

FIG. 1. (Color online) Experimental (red circles) and calculated (black line) diffraction patterns along with the difference profile (blue "a" line) of monoclinic OO phase II at $190 \mathrm{~K}$. Vertical bars indicate the calculated Bragg peak positions. Green "b" line corresponds to the face-centered-cubic OD phase I at $210 \mathrm{~K}$. Inset shows the cell volume versus temperature in the OO phase II. Dashed and dotted lines are linear regression to data above and below $T_{g}$. Vertical dashed line shows $T_{g}=130 \mathrm{~K}$.

difference between this perfectly translational and orientational ordered phase and the so-called plastic phase, for which orientational disorder (tumbling motion of the whole molecule) exists. Even more, the orientational disorder of the plastic phase is not necessarily characterized by a discrete number of distinguishable orientations (Frenkel model). Well-known results from incoherent quasielastic neutron scattering evidenced an (almost) isotropic tumbling motion (rotational diffusion model ${ }^{39}$ ) for many molecules in their plastic phases. ${ }^{40,41}$ In fact, the basis of the entropy criterion for the definition of a plastic phase (the melting entropy should be generally smaller than $5 R / 2, R$ being the universal gas

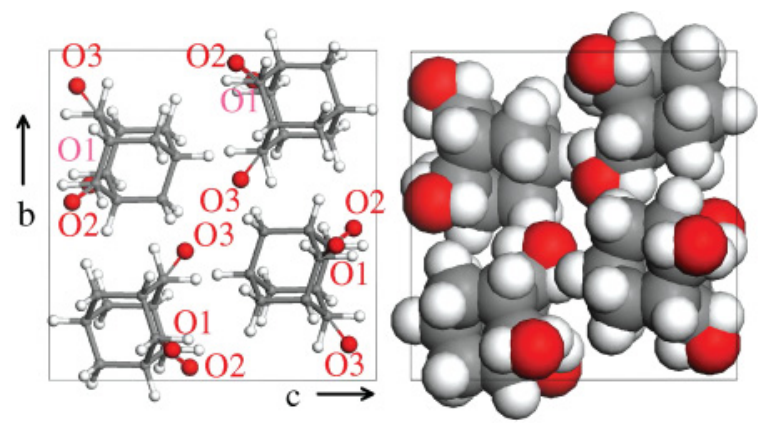

FIG. 2. (Color online) Left panel: Picture of the (001) crystallographic phase of OO phase II with oxygen atoms (red) within their three occupational sites. Red (darker gray in printed version) labeled atoms correspond to the oxygen atoms within their three sites of fractional occupancies $0.25(\mathrm{O} 1), 0.25(\mathrm{O} 2)$, and $0.50(\mathrm{O} 3)$. Right panel: The same projection with $70 \%$ of the van der Waals radii of the atoms.

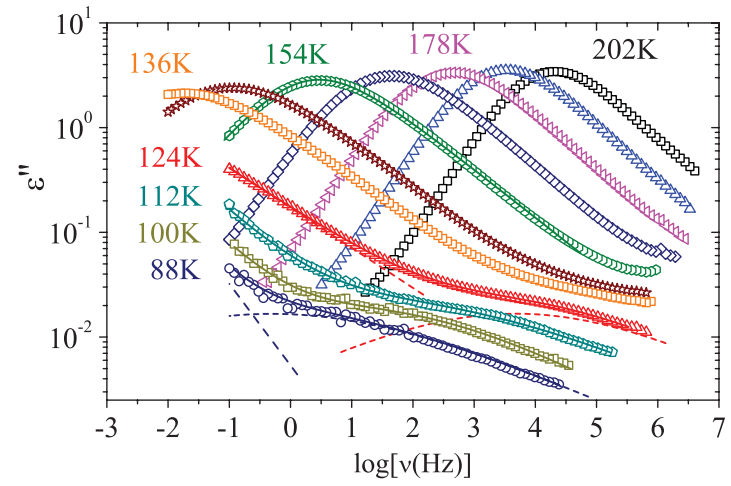

FIG. 3. (Color online) Dielectric loss spectra of phase II of 2adamantanone for several temperatures. The solid lines are examples of the fits using Havriliak-Negami and Cole-Cole functions for the $\alpha$ - and $\beta$-processes, respectively.

constant) ${ }^{42}$ certifies the similarities between the orientational disorder of liquid and plastic phases.

As a consequence of the three different molecular orientations in the low-temperature lattice, large-angle rotations around a threefold axis, which would exist for a molecule devoid of the oxygen atom, appear. These rotations promote an effective time-average fluctuation of the molecular dipole which causes a contribution to the dielectric susceptibility. Obviously, diffraction techniques cannot provide information about the dynamics of the dipole reorientation, but they highlight the disorder and quantify unequivocally the possible molecular orientations within the commensurate long-range lattice. It is important to note that, since the fractional occupancies of the three orientational sites have been shown to be constant in temperature, this means that a sort of local symmetry is preserved and that it can be assured only if correlation among motions of different molecules exists.

Figure 3 shows examples of the dielectric loss spectra of $2 \mathrm{~A}=\mathrm{O}$ for a set of temperatures in phase II. The $\alpha$-relaxation process is clearly accompanied by a secondary $\beta$-relaxation with a smaller strength $\left(\Delta \varepsilon_{\alpha} / \Delta \varepsilon_{\beta} \approx 500\right)$.

The dielectric loss spectra were fitted according to the Havriliak-Negami function with exponents $0.55<\alpha_{\mathrm{HN}}<$ 0.89 and $0.57<\beta_{\mathrm{HN}}<0.71$, corresponding to a quite small stretching exponent parameter $\beta_{\mathrm{KWW}}=0.50-0.57$ (calculated according to the Alvarez-Alegria-Colmenero equation ${ }^{43}$ ), while the $\beta$-relaxation appearing at low temperature and high frequency was described with a Cole-Cole function $\left(0.45<\alpha_{\mathrm{CC}}<0.57\right)$. It is noteworthy that the typical $\beta_{\mathrm{KWw}}$ of $\alpha$-relaxation in the OD phase I is about 0.9 (see upper inset of Fig. 4). The fact that in the ordered phase $\beta_{\mathrm{KWw}}$ is quite small is an indication that strong orientational correlations should exist between nearest neighbors. Such a fact is reinforced by the low value of the Kirkwood factor $g_{\mathrm{K}}$ (see lower inset of Fig. 4) which indicates a strong antiferroelectric order ${ }^{44}$ in phase II. The calculation of the Kirkwood correlation factor $g_{\mathrm{K}}$ has been obtained, as usual, through the following equation: ${ }^{32,45,46}$

$$
\begin{aligned}
\mu^{2} & =9 \varepsilon_{0} k_{\mathrm{B}} T \frac{V}{Z} \frac{\left(\varepsilon_{S}-\varepsilon_{\infty}\right)\left(2 \varepsilon_{S}+\varepsilon_{\infty}\right)}{\varepsilon_{S}\left(\varepsilon_{\infty}+2\right)^{2}}, \\
g_{\mathrm{K}} & =\frac{\mu^{2}}{\mu_{\text {gas }}^{2}},
\end{aligned}
$$




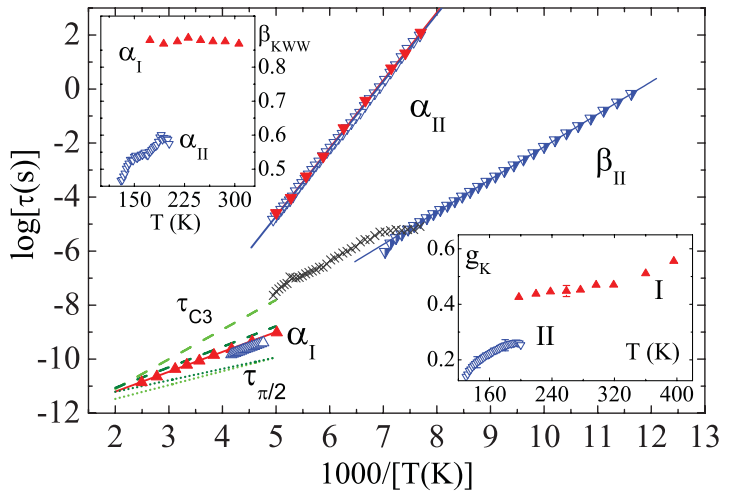

FIG. 4. (Color online) Relaxation times as a function of reciprocal of temperature for the different relaxation processes in the OD phase I and in the OO phase II. Phase I: Dashed lines show $\tau_{C 3}$, $2 \pi / 3$-rotations of the $\mathrm{C}=\mathrm{O}$ dipolar axis between the $\langle 001\rangle$ cubic lattice directions; dotted lines show $\tau_{\pi / 2}, \pi / 2$ jump rotations of the molecules around the $\mathrm{C}=\mathrm{O}$ axis; data are from NMR (Ref. 35) (dark green color) and from IQNS (Ref. 34) (light green color); $\alpha_{\text {I }}$ process (equivalent to rotations of the $\mathrm{C}=\mathrm{O}$ dipolar axis) from dielectric spectroscopy from Ref. 23 (red solid up triangles) and from this work (blue open up triangles). Phase II: $\alpha$-relaxation times are represented by open and closed inverted triangles, respectively, from this work and Ref. 23 (blue and red symbols, respectively), while $\beta$-relaxation times are shown as blue-white inverted triangles. Lines are linear regressions to data. Gray asterisks are the predicted $\beta$-relaxation times calculated according to the coupling model (see text). Inset: $\beta_{\mathrm{KWw}}$ (upper) and Kirkwood factor $g_{\mathrm{K}}$ (lower) of phases I and II as a function of temperature.

where $\mu_{\mathrm{gas}}^{2}$ is the dipole moment estimated in gas phase (equal to $3.4 \mathrm{D})^{32,33}, \varepsilon_{0}$ is the vacuum permittivity, $\varepsilon_{S}$ and $\varepsilon_{\infty}$ are the static and relaxed permittivity (obtained from dielectric spectra), and the parameter $V / Z$ is the molecular cell volume as obtained from $x$-ray diffraction (see inset in Fig. 1). It should be mentioned that the value of the $g_{\mathrm{K}}$ factor should be taken cautiously because it has been calculated assuming spatial isotropy. Such an assumption can be taken over for liquids or translationally ordered cubic phases, but it is just a first approximation for anisotropic media, as the orthorhombic $\mathrm{OO}$ phase II. Nevertheless, it still provides a good figure to account for the changes with temperature and for a rough comparison with the isotropic OD phase I.

Figure 4 collects the relaxation time for both processes as well as results previously published for the relaxation times in OD phase I obtained from different techniques. ${ }^{23,32-35}$ Relaxation times for all the different processes follow Arrhenius temperature behaviors. It is noteworthy that activation energy for the $\alpha$-process in phase II is higher $\left[E\left(\alpha_{\mathrm{II}}\right)=\right.$ $5746 \mathrm{~K}$ ] than that in the OD phase I $\left[E\left(\alpha_{\mathrm{I}}\right)=1699 \mathrm{~K}\right]$, as an obvious consequence of the stronger interaction in the former phase that cannot be merely explained by the small volume change at the transition $\left(\Delta V_{\mathrm{II} \rightarrow \mathrm{I}} / V_{\mathrm{I}} \approx 3 \%\right)$. This change goes along the value of the prefactor Arrhenius times, $\tau_{\infty}\left(\alpha_{\mathrm{I}}\right)=1.25 \times 10^{-13} \mathrm{~s}$, while $\tau_{\infty}\left(\alpha_{\mathrm{II}}\right)=6.45 \times 10^{-18} \mathrm{~s}$, an unphysical value for a noncooperative process. These values perfectly agree with those previously published..$^{23}$ Moreover, the $\alpha$-relaxation time reaches $10^{2} \mathrm{~s}$, the conventional relaxation time for glass transition, at $T_{g}=130 \mathrm{~K}$, coincident with the region where a change of slope of volume cell versus temperature is reported (see inset in Fig. 1), another indication of glassy-like behavior. Additional arguments support the aforementioned cooperativity of the $\alpha$-process in the $\mathrm{OO}$ phase II. By inspecting the structural arrangement of the molecules in the phase II with the associated van der Waals radii (right panel of Fig. 2) it becomes clear that there is no way for the molecules to jump from one orientational site to another without intermolecular cooperation and correlated motions of adjacent molecules. Moreover, since the fractional occupancies of the three sites have been shown to be constant in temperature, this means that a sort of local symmetry is preserved and that it can be assured only if correlation among molecules exists, as results from the previously discussed low values of $g_{\mathrm{K}}$ and $\beta_{\mathrm{KWw}}$. The cooperative character of the $\alpha$ relaxation is commonly associated with the nonexponentiality, described by the stretching $\beta_{\mathrm{KWw}}$ parameter ${ }^{47}$ due to the many-body coupling ${ }^{48}$ or to dynamic heterogeneities. ${ }^{49,50}$ On crossing from phase I to phase II around $200 \mathrm{~K}$, the stretching parameter suddenly changes from values close to 1 (indicating Debye behavior) to less than 0.6 and, on further cooling within phase II, the value still reduces to less than 0.5 at $T_{g}$ and this is an effect to be ascribed to the cooperativity of many-body molecular motions. A nonexponential relaxation or a broad loss peak as a consequence of the addition of different distinct noncooperative modes with distinct energy barriers must be discarded in the present case due to the conflict with temperature variation of the relaxation shape. As has been shown by Zorn, ${ }^{51}$ in that case the standard deviation of distribution of logarithmic relaxation times, $\sigma_{\ln \tau}$, related to the variance of the distribution of activation energy, $\sigma_{\mathrm{E}}^{2}$, according to the law $\sigma_{\ln \tau}=\sigma_{\mathrm{E}} / \mathrm{k}_{\mathrm{B}} \mathrm{T}$, should be proportional to the width of the peak loss. Then, if the distribution of activation energy is constant with temperature (as should be expected in the present case), the width should be proportional to reciprocal temperature. Figure 5 shows $\sigma_{\ln \tau}$ as a function of the reciprocal

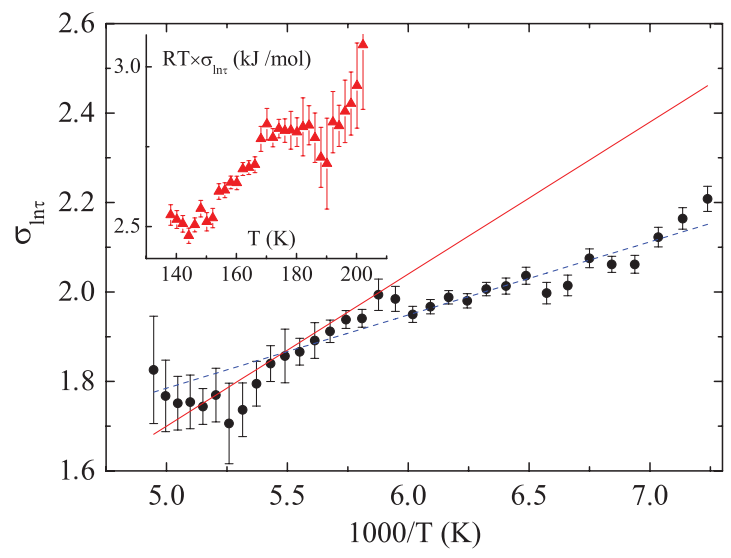

FIG. 5. (Color online) Standard deviation of distribution of logarithmic relaxation times, $\sigma_{\ln \tau}$, as a function proportional to reciprocal temperature obtained by means of the procedure described in Ref. 51 through the fitting parameters obtained from experimental values (filled circles). Continuous red line would correspond to a constant distribution of activation energy $\left(\sigma_{\mathrm{E}}\right)$ barriers while dotted blue line represents the fit of the obtained values. Inset: Distribution of activation energy, $\sigma_{\mathrm{E}}$, obtained by means of $\sigma_{\mathrm{E}}=\mathrm{RT} \sigma_{\ln \tau}$ as a function of temperature. 
temperature (from the $\mathrm{OO}$ to $\mathrm{OD}$ phase transition to the glass transition) obtained by means of Zorn's work through the experimental fitted parameters. It clearly shows that the measured width is much narrower than the value that would be expected and thus that the distribution of activation energy $\sigma_{\mathrm{E}}$ is not constant, thus disregarding the possibility of several relaxing modes with distinct energy barriers.

As for the relaxation time of the $\beta$-process, data in Fig. 4 provide an Arrhenius behavior with $E\left(\beta_{\mathrm{II}}\right)=2744 \mathrm{~K}$ and $\tau_{\infty}\left(\beta_{\mathrm{II}}\right)=6.53 \times 10^{-15} \mathrm{~s}$. The good agreement between the rotational dynamics displayed in phase I by the $\alpha$-process (a motion with a very small degree of cooperativity) and the extrapolation of the temperature behavior of the $\beta$-process at higher temperature is noteworthy.

It is well known that Johari-Goldstein secondary $\beta$ relaxation processes appear in OD phases. ${ }^{16}$ In some cases, the question of the appearance of the so-called excess wing of the $\alpha$-relaxation for this kind of material is still a matter of controversy, ${ }^{16}$ and it is not even excluded that excess wing and $\beta$-relaxation are expressions of the same microscopic mechanism. Nevertheless, as far as we know, there are few experiments reporting the existence of relaxation processes in OG from orientationally ordered phases and only one showing two relaxation processes:

(i) Johari ${ }^{52,53}$ reported the existence of only one, simply activated, almost Debye process in $\mathrm{CuCN}$ OG, giving rise to a specific-heat effect due to the freezing at $T_{g}$, and ascribing it to thermally activated motion of $\mathrm{C}$ and $\mathrm{N}$ atoms orienting the - $\mathrm{Cu}-\mathrm{C}-\mathrm{N}$ chains in different possible configurations or to the diffusion of vacancies in the disordered arrangement.

(ii) In the low-temperature crystal phase of $\mathrm{TINO}_{2}$ (Ref. 54) the molecular dynamics is also controlled only by one relaxation process with Arrhenius energy, giving rise at $T_{g}$ to glassylike behavior of specific heat, expansivity, and temperature derivative of the mean-square atomic displacement. Such behavior was explained in terms of anharmonicity-induced change of the $\mathrm{NO}_{2}^{-}$dipole vector when a vacancy diffuses to its near-neighbor lattice site.

(iii) For halogenomethane compounds $\left(\mathrm{CBr}_{n} \mathrm{Cl}_{4-n}, n=0,1\right.$, 2) the existence of two relaxations was explained by means of the different environment of the two sets of molecules (formed by one and by three molecules) of the asymmetric unit $\left(Z^{\prime}=\right.$ 4). ${ }^{27,55}$

In the present system the statistical disorder due to the fractional occupancy of the oxygen atom of the only molecule in the asymmetric unit of phase II gives rise to a clear primary relaxation with a considerable strength (see Fig. 3). As for the $\beta$-relaxation, which has all the properties of an original JG $\beta$-relaxation, the origin cannot be attributed to the existence of different independent molecules (as $Z^{\prime}=1$ ), as in the case of halogen-methane compounds. Moreover, the rigid $\mathrm{C}=\mathrm{O}$ group attached to the adamantane skeleton rules out the existence of intramolecular dipole fluctuations ${ }^{23,56-58}$ as the origin of the secondary relaxation. If $\beta$-relaxation is thus related to some intermolecular mechanism and only one kind of molecule is present in the asymmetric unit, it would then mean that the $\beta$-relaxation should be linked with the physically well identified $\alpha$-relaxation, i.e., dipole fluctuations due to the oxygen site disorder. Only a few theoretical models assume explicitly the correlation between $\alpha$ - and JG $\beta$-relaxation to account globally for glass-forming dynamics. ${ }^{19,59,60}$ According to the point of view of the coupling model $(\mathrm{CM}),{ }^{5,19}$ the $\beta$-process is the primitive motion which initiates the cooperative $\alpha$-process at particular sites in the disordered structure. The more cooperative the $\alpha$-process is, the greater is the time-scale separation from the JG $\beta$-process. The cooperativity of the $\alpha$-process reflects into a broader distribution of relaxation times, i.e., into a small $\beta_{\mathrm{KWw}}$. The CM gives a quantitative relation, due to many-molecule dynamics, between the primitive $\left(\tau_{P}\right) \mathrm{JG}$ relaxation time (or $\left.\tau_{\mathrm{JG}}\right)$ and $\tau_{\alpha}$ :

$$
\tau_{\mathrm{JG}} \approx \tau_{P}=\tau_{\alpha}^{\beta_{\mathrm{KWw}}} t_{c}^{1-\beta_{\mathrm{Kww}}},
$$

where $t_{c}$ is the crossover time where the many-body dynamics start to occur ( $2 \mathrm{ps}$ in molecular glass formers). In the case of $2 \mathrm{~A}=\mathrm{O}$, the calculated primitive $\tau_{\mathrm{JG}}$ matches exactly the relaxation time for the $\beta$-process (gray asterisks in Fig. 4) within the temperature range for which available $\beta_{\mathrm{KWW}}$ data for the $\alpha$-process exist.

So, whereas in the OD phase the $\alpha$-relaxation could occur without strong interactions among the neighbors, $\beta_{\mathrm{KWw}}$ is high, and the JG and $\alpha$-processes are not differentiated in time scale, in the $\mathrm{OO}$ phase, which has less volume and restricted transitions, the $\alpha$-process has to occur with a high degree of cooperativity. This goes along the highest activation energy and the smaller (unphysical) prefactor $\tau_{\infty}(\alpha) \approx 10^{-17} \mathrm{~s}$ characteristic for this process, a sort of "compensation law" already shown for several systems with correlated motions. ${ }^{61,62}$ Moreover, Eq. (2) predicts that the ratio between the activation energy of $\alpha$ - and JG processes should be equal to $\beta_{\mathrm{KWw}}$, as in our case. The fact that the calculated primitive relaxation time through Eq. (2) matches exactly the $\beta$-relaxation time is another direct indication of the cooperativity of the $\alpha$ relaxation process.

An alternative universal origin for the $\beta$-process was recently proposed in the framework of random first order theory (RFOT). ${ }^{22}$ Within this theory, primary relaxation occurs through reconfiguration of activated events involving compact and correlated regions, while secondary relaxation is governed by string-like or ramified events, dominating the low-barrier tail of the activation-energy distribution. This last kind of process dominates the high-temperature regime and merges with the primary one but, on cooling, the timescale and the energy barrier of the primary relaxation become larger and larger and separate. The relative intensity of secondary over primary relaxation is predicted to be more significant for fragile than for strong systems, and this is in agreement with our case, where in the merging region the JG $\beta$-relaxation intensity is much smaller than the $\alpha$ one. An application of this model to our case is tempting: The compact region could be identified with correlated domains of adjacent $2 \mathrm{~A}=\mathrm{O}$ molecules that reorient themselves in a cooperative way and the secondary relaxation with some independent motions of isolated and rare defects at the border of the correlated regions. Nevertheless, no quantitative prediction can be obtained from the RFOT model making impossible an explicit test for our case. Further developments of the RFOT, taking into account a possible relation between timescale separation between $\alpha$ and $\beta$-relaxation on one side and the width of $\alpha$-relaxation time distribution on the other side, could help to clarify 
the universal mechanism of secondary relaxation, and are therefore welcome.

\section{CONCLUSIONS}

In conclusion, the lattice structure of phase II of 2adamantanone shows the existence of a statistical disorder due to the fractional occupancy of the oxygen atom of the molecule which creates strongly correlated large-angle dipole fluctuations making possible the appearance of $\alpha$-relaxation. In addition, the coupling model is able to explain the pure Johari-Goldstein $\beta$-relaxation emerging in such ordered phase through a low stretching $\beta_{\mathrm{KWW}}$ value (i.e., high coupling parameter, $\left.1-\beta_{\mathrm{KWW}}\right)$ in spite of the strong (Arrhenius) character of the $\alpha$-relaxation process. The emergence of a glassy-like $\alpha$ - $\beta$ dynamics even in an "ordered" system where coupling exists between motions on different time and length scales is a relevant result for studies of the glass transition. It is noteworthy that a recent theoretical model, ${ }^{63}$ based on $s-p$ spherical spin systems, has been able to display two-time-scale bifurcation and to show the interrelation of dynamic processes active on separated time scales, with a good qualitative agreement with the predictions of Eq. (2). Systems similar to ours, with definite degrees of disorder and well-known molecular interactions, are good candidates for theoretical models and numerical simulations.

\section{ACKNOWLEDGMENTS}

This work was supported by the Spanish Ministry of Science and Innovation (Grant No. FIS2011-24439) and the Catalan Government (Grant No. 2009SGR-1251). One of us (S.C.) thanks the Spanish Ministry (SB2009-0139) for an invited position at the Universitat Politècnica de Catalunya.

*jose.luis.tamarit@upc.edu

${ }^{1}$ C. A. Angell, K. L. Ngai, G. B. McKenna, P. F. McMillan, and

S. W. Martin, J. Appl. Phys. 88, 3113 (2000).

${ }^{2}$ P. G. Debenedetti and F. H. Stillinger, Nature (London) 410, 259 (2001).

${ }^{3}$ L. Berthier and G. Biroli, Rev. Mod. Phys. 83, 587 (2011).

${ }^{4}$ G. P. Johari and M. Goldstein, J. Chem. Phys. 53, 2372 (1970).

${ }^{5}$ K. L. Ngai, Phys. Rev. E 57, 7346 (1998).

${ }^{6}$ U. Schneider, R. Brand, P. Lunkenheimer, and A. Loidl, Phys. Rev. Lett. 84, 5560 (2000).

${ }^{7}$ G. P. Johari, G. Power, and J. K. Vij, J. Chem. Phys. 116, 5908 (2002).

${ }^{8}$ K. L. Ngai and M. Paluch, J. Chem. Phys. 120, 857 (2004).

${ }^{9}$ T. Blochowicz and E. A. Rössler, Phys. Rev. Lett. 92, 225701 (2004).

${ }^{10}$ R. Böhmer, G. Diezemann, B. Geil, G. Hinze, A. Nowaczyk, and M. Winterlich, Phys. Rev. Lett. 97, 135701 (2006).

${ }^{11}$ R. Casalini and C. M. Roland, Phys. Rev. Lett. 102, 035701 (2009).

${ }^{12}$ G. P. Johari, J. Chem. Phys. 58, 1766 (1973).

${ }^{13}$ A. Döß, M. Paluch, H. Sillescu, and G. Hinze, Phys. Rev. Lett. 88, 095701 (2002).

${ }^{14}$ M. Vogel and E. Rössler, J. Phys. Chem. B 104, 4285 (2000); M. Vogel and E. Rössler, J. Chem. Phys. 114, 5802 (2001).

${ }^{15}$ C. A. Angell, Science 267, 1924 (1995).

${ }^{16}$ R. Brand, P. Lunkenheimer, U. Schneider, and A. Loidl, Phys. Rev. Lett. 82, 1951 (1999).

${ }^{17}$ S. Hensel-Bielowka and M. Paluch, Phys. Rev. Lett. 89, 025704 (2002).

${ }^{18}$ P. Lunkenheimer, R. Wehn, Th. Riegger, and A. Loidl, J. Non-Cryst. Solids 307-310, 336 (2002).

${ }^{19}$ K. L. Ngai, J. Phys.: Condens. Matter 15, S1107 (2003).

${ }^{20}$ H. Tanaka, J. Chem. Phys. 111, 3175 (1999).

${ }^{21}$ H. Tanaka, Phys. Rev. E 69, 021502 (2004).

${ }^{22}$ J. D. Stevenson and P. G. Wolynes, Nature Phys. 6, 62 (2010).

${ }^{23}$ R. Brand, P. Lunkenheimer, and A. Loidl, J. Chem. Phys. 116, 10386 (2002).

${ }^{24}$ A. Drozd-Rzoska, S. J. Rzoska, S. Pawlus, and J. Ll. Tamarit, Phys. Rev. B 73, 224205 (2006).

${ }^{25}$ M. A. Ramos, S. Vieira, F. J. Bermejo, J. Dawidowski, H. E. Fischer, H. Schober, M. A. González, C. K. Loong, and D. L. Price, Phys. Rev. Lett. 78, 82 (1997)

${ }^{26}$ H. E. Fischer, F. J. Bermejo, G. J. Cuello, M. T. Fernández-Diaz, J. Dawidowski, M. A. González, H. Schober, and M. Jimenez-Ruiz, Phys. Rev. Lett. 82, 1193 (1999).

${ }^{27}$ M. Zuriaga, L. C. Pardo, P. Lunkenheimer, J. Ll. Tamarit, N. Veglio, M. Barrio, F. J. Bermejo, and A. Loidl, Phys. Rev. Lett. 103, 075701 (2009).

${ }^{28}$ Y. Huang, D. F. R. Gilson, I. S. Butler, and F. Morin, J. Phys. Chem. 95, 2151 (1991).

${ }^{29}$ N. Arul Murugan, R. S. Rao, S. Yashonath, S. Ramasesha, and B. K. Godwal, J. Phys. Chem. B 109, 17296 (2005).

${ }^{30}$ J. P. Amoureux, M. Bee, and J. C. Damien, Acta Cryst. B 36, 2633(1980).

${ }^{31}$ N. Arul Murugan and S. Yashonath, J. Phys. Chem. B 109, 2014 (2005).

${ }^{32}$ J. P. Amoreux, M. Sahour, C. Fernandez, and P. Bodart, Phys. Status Solidi 143, 441 (1994).

${ }^{33}$ J. P. Amoureux, M. Castelain, M. Bee, B. Arnaud, and M. L. Shouteeten, J. Phys. C: Solid State Phys. 15, 1319 (1982).

${ }^{34}$ M. Bee and J. Amoreux, Mol. Phys. 47, 533 (1982).

${ }^{35}$ R. Decressain, J.P. Amoreux, and E. Cochon, Phys. Status Solidi 190, 295 (1995).

${ }^{36} \mathrm{Ph}$. Negrier, M. Barrio, J. Ll. Tamarit, N. Veglio, and D. Mondieig, Cryst. Growth Design 10, 2793 (2011).

${ }^{37}$ J. N. Sherwood, The Plastically Crystalline State: Orientationally Disordered Crystals (Wiley, New York, 1979).

${ }^{38} \mathrm{M}$. Foulon, T. Belgrand, C. Gors, and M. More, Acta Cryst. B 45, 404 (1989).

${ }^{39}$ M. Bée, Quasielastic Neutron Scattering (Adam Hilger, Bristol, 1988).

${ }^{40}$ J. C. Frost, A. J. Leadbetter, and R. M. Richardson, Philos. Trans. R. Soc. London B 290, 567 (1980).

${ }^{41}$ A. J. Leadbetter, R. C. Ward, and R. M. Richardson, J. Chem. Soc., Faraday Trans. 2 81, 1067 (1985).

${ }^{42}$ J. Timmermans, J. Phys. Chem. Solids 18, 1 (1961). 
${ }^{43}$ F. Alvarez, A. Alegría, and J. Colmenero, Phys. Rev. B 44, 7306 (1991).

${ }^{44}$ J. C. Martinez-Garcia, J. L1. Tamarit, S. Capaccioli, M. Barrio, N. Veglio, and L. C. Pardo, J. Chem. Phys. 132, 164516 (2010).

${ }^{45}$ C. J. F. Böttcher, Theory of Electric Polarization, Vol. 1: Dielectrics in Static Fields (Elsevier, Amsterdam, 1973).

${ }^{46} \mathrm{~F}$. Kremer and A. Schonhals, Broadband Dielectric Spectroscopy (Springer Verlag, Berlin, 2003).

${ }^{47}$ R. Bohmer, K. L. Ngai, C. A. Angell, and D. J. Plazek, J. Chem. Phys. 99, 4201 (1993).

${ }^{48}$ K. L. Ngai, J. Phys.: Condens. Matter 11, A119 (1999).

${ }^{49}$ M. D. Ediger, Annu. Rev. Phys. Chem. 51, 99 (2000).

${ }^{50}$ R. Richert, J. Phys.: Condens. Matter 14, R703 (2002).

${ }^{51}$ R. Zorn, J. Chem. Phys. 116, 3204 (2002).

${ }^{52}$ J. Wang, M. F. Collins, and G. P. Johari, Phys. Rev. B 65, 180103R (2002).

${ }^{53}$ J. Wang and G. P. Johari, Phys. Rev. B 68, 214201 (2003).
${ }^{54}$ G. P. Johari, M. F. Collins, and R. Feyerherm, Phys. Rev. B 61, 5827 (2000).

${ }^{55}$ M. Zuriaga, M. Carignano, and P. Serra, J. Chem. Phys. 135, 044504 (2011).

${ }^{56}$ P. D. Harvey, I. S. Butler, D. F. R. Gilson, and P. T. T. Wong, J. Phys. Chem. 90, 4546 (1986).

${ }^{57}$ L. Bistricic, G. Baranovic, and K. Mlinaric-Majerski, Spectr. Acta A 54, 1961 (1998).

${ }^{58}$ L. Bistricic, G. Baranovic, and V. Volovsek, J. Mol. Struct. 482-483, 661 (1999).

${ }^{59}$ J. Y. Cavaille, J. Perez, and G. P. Johari, Phys. Rev. B 39, 2411 (1989).

${ }^{60}$ G. Diezemann, U. Mohanty, and I. Oppenheim, Phys. Rev. E 59, 2067 (1999).

${ }^{61}$ A. Yelon and B. Movaghar, Phys. Rev. Lett. 65, 618 (1990).

${ }^{62}$ K. L. Ngai and A. Schönhals, J. Polym. Sci. Part B 36, 1927 (1998).

${ }^{63}$ A. Crisanti, L. Leuzzi, and M. Paoluzzi, Eur. Phys. J. E 34, 98 (2011). 